\title{
Automatic segmentation of large bowl obstruction area with Hough transform from erect abdominal radiograph images
}

\author{
Kwang Baek Kim¹, Doo Heon Song', Young Woon Woo ${ }^{3}$ \\ ${ }^{1}$ Division of Computer Software Engineering, Silla University, Busan, Republic of Korea \\ ${ }^{2}$ Department of Computer Games, Yong-In SongDam College, Yong-in, Republic of Korea \\ ${ }^{3}$ Department of Applied Software Engineering, Dong-eui University, Busan, Republic of Korea
}

\section{Article Info \\ Article history: \\ Received Jul 31, 2020 \\ Revised Sep 22, 2020 \\ Accepted Oct 9, 2020 \\ Keywords: \\ Automatic segmentation \\ Canny edge \\ Erect radiograph \\ Hough transform \\ Large bowl obstruction}

\begin{abstract}
Large bowel obstruction is less frequent but often appears acute and needs emergent treatment. Erect abdominal radiograph is usually the first imaging study performed in patients suspected of having large bowel obstruction. However, that modality suffers from operator subjectivity thus a fully automatic computer aided tool is necessary. In this paper, we propose an automatic large bowel feature (air-fluid region) segmentation method based on Canny edge detection and Hough transform. In experiment, the proposed method was successful in finding target region from large bowel obstruction patients' radiographic images in all 30 cases provided. While limited only applicable to the large bowel obstruction cases, the proposed method is practically feasible in application.
\end{abstract}

This is an open access article under the CC BY-SA license.

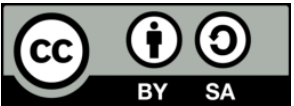

\section{Corresponding Author:}

Kwang Baek Kim

Division of Computer Software Engineering

Silla University

Busan 617-736, Korea

Email: gbkim@silla.ac.kr

\section{INTRODUCTION}

Bowel obstruction or intestinal obstruction is a mechanical or functional obstruction of the intestines which prevents the normal movement of the products of digestion. Signs and symptoms include abdominal pain, vomiting, bloating and not passing gas [1]. Large-bowel obstruction (LBO) differs substantially from small bowel obstruction (SBO). LBO constitutes $20 \%$ of all mechanical obstruction. Its etiology is agedependent with colonic cancer being the most common cause in adults [2]. Patients with LBO are usually elderly, and the signs and symptoms of LBO are often insidious in contrast to the abrupt onset of symptoms seen in most SBO [3] and the causes of LBO and SBO differ substantially [4]. While LBO may develop over a protracted period and less frequently occurred than SBO in general, the clinical presentation is often acute and includes abdominal pain, constipation or obstipation, and abdominal distension [5]. Emergency surgery or colonoscopy is usually required to relieve the obstruction [6]. Thus, treating acute LBO is particularly important due to its high morbidity and mortality rates if left untreated [7].

Image analysis plays a vital role in the management of LBO by identifying the location, degree, and cause of obstruction. Abdominal radiography is usually the first imaging study performed in patients suspected of having LBO. While the reported sensitivity of abdominal radiography for the detection of LBO is similar to that for the detection of SBO (84\% vs. $82 \%$, respectively), the reported specificity is considerably different ( $72 \%$ vs. $83 \%$, respectively) and as a result, it may be difficult to distinguish between obstruction and colonic pseudo-obstruction in a patient with a distended colon [8, 9]. Furthermore, 
radiographic studies showed limited capability in the diagnosis of bowel infections [10-12]. Thus, further evaluation with multidetector computed tomography (MDCT) has become the standard of care to identify the site, severity, and etiology of obstruction $[13,14]$. However, this modality is expensive, time consuming, and associated with high radiation exposure that is over 10 times more than the abdominal radiography [15].

In sum, the diagnostic value of abdominal radiographs is limited but continues to be recommended as an important component of the diagnostic imaging pathway of acute abdomen due to above-mentioned trade-off [16]. Normal colonic caliber is variable due to its high distensibility, and for practical purpose cecum $>9 \mathrm{~cm}$ and rest of the colon $>6 \mathrm{~cm}$ in diameter is considered as dilated [6]. The presence of colonic air fluid levels usually indicates an acute mechanical obstruction. The absence of distal rectal gas is not a reliable sign of LBO as it can be seen in colonic ileus as well. However, it is difficult to evaluate all the loops or make an etiologic diagnosis with radiographic image analysis alone. Moreover, it is operator dependent and difficult to perform in acute state as well as in obese patient [17].

In this paper, we propose a fully automatic segmentation method for LBO structure detection. Segmentation process is inevitable procedure for making stable diagnosis by medical image analysis but frequently has been a source of operator subjectivity especially using ultrasound or plain radiography [18, 19]. In LBO radiographic image analysis, we may use either supine and erect (or left lateral decubitus). It is reported that erect radiography not only gave slightly better performance in diagnostic accuracy and the intra-rater and inter-rater agreement but also air-fluid levels were only seen on the erect radiograph image [20]. Moreover, for LBO, we can use morphological information such as the discriminative shape of air fluid level from erect radiograph. Still, doctors with less experience may find it difficult to interpret abdominal radiographs that appear to have normal anatomy but have an unusual bowel gas pattern [21]. Thus, automatic segmentation procedure gives less chance of making incorrect decision in diagnosis. Automatic segmentation process includes many image processing steps such as binarization and noise filtering. Our proposed method relies on traditional edge analysis and Hough transform [22] as the main tools to locate the air-fluid area from erect LBO radiographs correctly.

\section{METHOD}

\subsection{Preprocessing and canny edge processing}

A typical erect abdominal radiograph having LBO is shown as Figure 1. The lung is shown as relatively low intensity and located in the upper part of the body, and the stomach is in the upper right of the body with low intensity with horizontal flatness. The pelvis exists in the lower part of the body and with relatively high intensity. The intestine which is our region of interest (ROI) is located between the lungs and the pelvis.

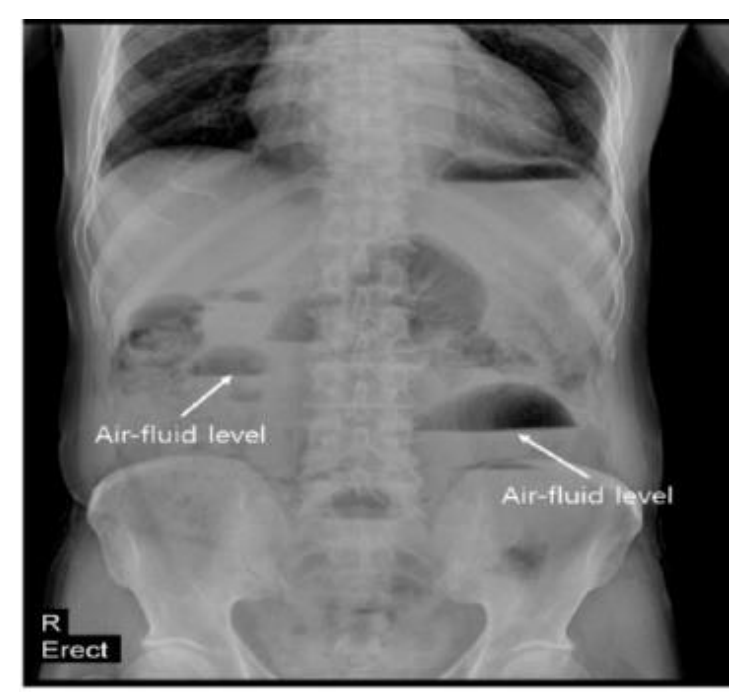

Figure 1. Typical erect abdominal radiograph with LBO

The target area of our method is the semicircular shape of air-fluid area in the lower boundary of the intestinal obstruction area appearing in the LBO patient's radiographic image as shown in Figure 1. To locate the target area, we apply canny edge detection algorithm [23]. Edge detection is the process of finding sharp separations in an image. The separations are significant changes in pixel intensity which distinguish barriers 
of objects in a scene. From the input radiographic image shown as Figure 2(a), we first apply Gaussian Blur mask to filter noises and obtain images like Figure 2(b). The second step is to estimate gradients. Calculation of the gradient is done by the Sobel kernel which is applied to each pixel in the image in both directions. Strength of the edge is determined by gradient magnitude while direction of the gradient is used to determine for each pixel the direction with the largest change in intensity and we obtain the intermediate result as shown in Figure 2(c).

The gradient direction needs to be rounded to nearest 45-degree step. Only the pixel with maximum edge strength will be preserved, otherwise it will be suppressed through a process on nonmaximum suppression for edge thinning. After that, we apply double thresholding technique to remove noises and determine edges from the image. Weak edges are finally preserved or suppressed using edge tracking by hysteresis and finally we obtain edges shown as Figure 2(d). After Canny edge detection, we cut off upper part of the lung and lower part of the pelvis to restrict our ROI as the area having LBO structure to make our method more efficient in computation.

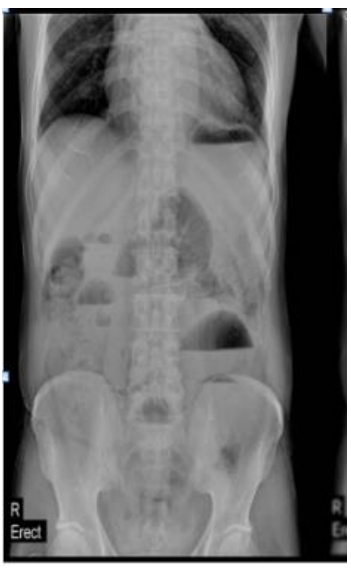

(a)

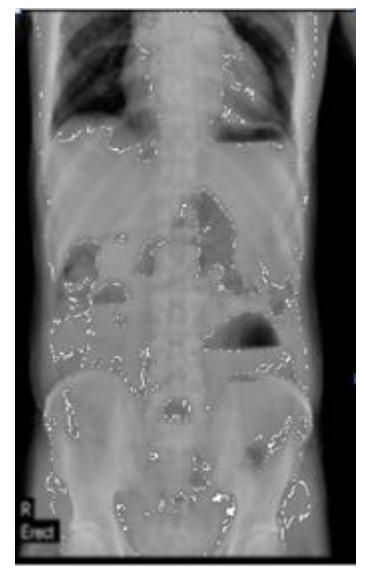

(b)

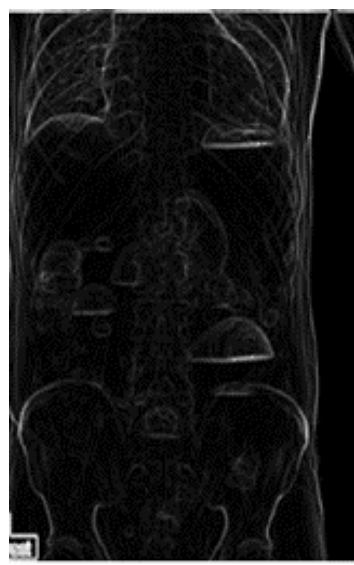

(c)

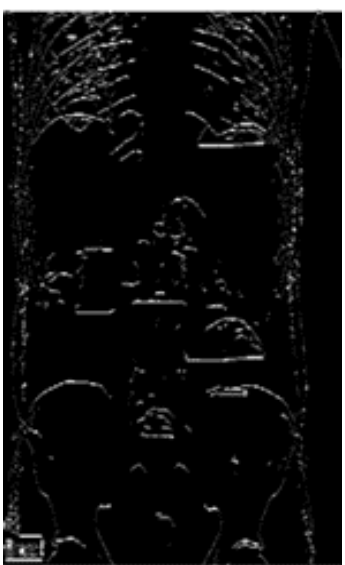

(d)

Figure 2. Canny edge application, (a) input, (b) after Gaussian blur, (c) after gradient, (d) extracted edges

\subsection{Extracting LBO features by Hough transform}

The Hough transform is a feature extraction technique used in image analysis, computer vision, and digital image processing [23-27]. The purpose of the technique is to find imperfect instances of objects within a certain class of shapes by a voting procedure for the possible geometric shapes of edges. The Hough transform can be defined as the transformation of a point in Cartesian space into the parameter space defined by the shape of the object of the interest. For any point (x, y) on a straight line (edge) can be transformed to $(\mathrm{r}, \theta)$ space called as Hough space by (1).

$$
r=x \cdot \cos (\theta)+y \cdot \sin (\theta)
$$

One way of interpreting this is to drop a perpendicular from the origin to the line. $\theta$ is the angle that the perpendicular makes with the $\mathrm{x}$-axis and $\mathrm{r}$ is the length of the perpendicular. $\theta$ is bounded by $[0,2 \pi]$ and $\mathrm{r}$ is bounded by the diagonal of the image. In this paper $\theta$ is limited to $[87,93]$ in degree.

To detect an accurate straight line, in this paper, the average of the extracted edge lengths is set as the threshold and the number of sine curves passing through a point appearing on the Hough space is compared with the threshold, The point with the most intersection in that process is chosen to be transformed into a straight line of (x, y) plane by applying (2). The result is shown as Figure 3(b).

$$
\begin{aligned}
& \sum_{y=0}^{H} x=\frac{(r-y \cdot \sin (\theta))}{\cos (\theta)} \\
& \sum_{x=0}^{W} y=\frac{(r-x \cdot \cos (\theta))}{\sin (\theta)}
\end{aligned}
$$

where $\mathrm{H}$ and $\mathrm{W}$ denote the height and width of the given image respectively. 
By taking the most intersected edges with Hough transformed lines from Figure 3(a) are chosen as candidates of the LBO segmentation features as shown in Figure 4(a). Then, we consider the morphological characteristic of LBO to locate the delated air fluid region as shown in Figure 4(b).

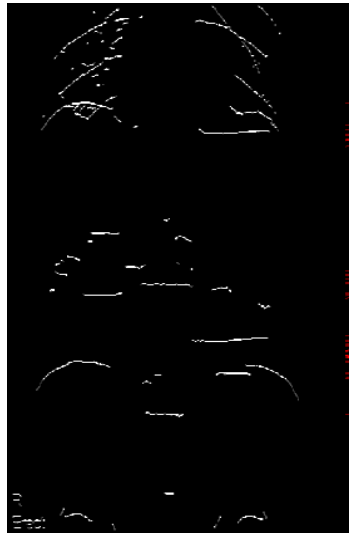

(a)

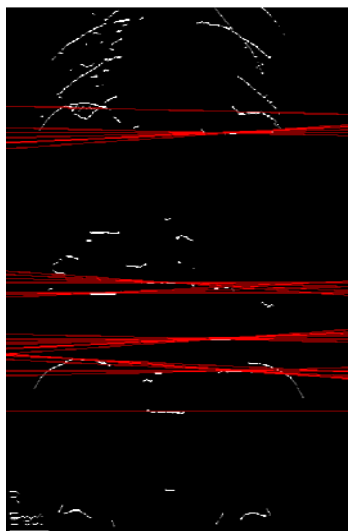

(b)

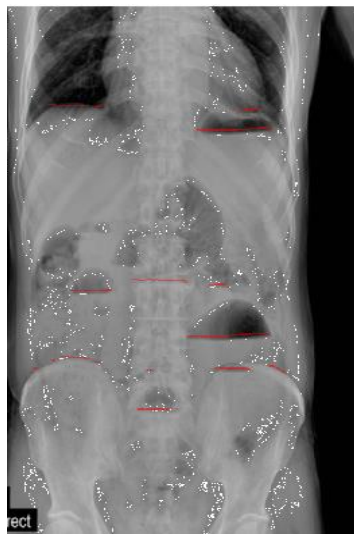

(a)

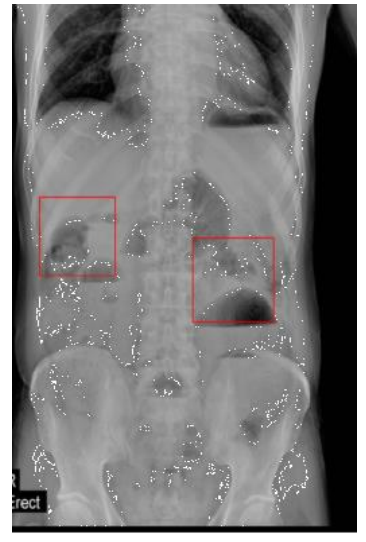

(b)

Figure 3. Effect of Hough transform, (a) canny edge result, (b) after Hough transform

Figure 4. Extracting LBO features example, (a) LBO feature candidates, (b) LBO features

\section{RESULTS}

The implementation of the proposed method is written by C\# under Visual Studio2015 environment on IBM compatible PC with Intel(R) Core (TM) i7-7500 CPU @ 1.80GHz and 16 GB RAM. An experiment is designed to verify of the proposed method finds LBO features from the erect radiograph image photographed by DK Medical Accuray-650R diagnostic radiation generator. Total 30 LBO patients' images are obtained from Baik Hospital, Busan, Korea. In this experiment, the proposed method was successful to locate the LBO features in all 30 input images. The performance of the implemented software was verified by the pathologists from Baik Hospital. An example of real-world LBO feature segmentation result is demonstrated in Figure 5.

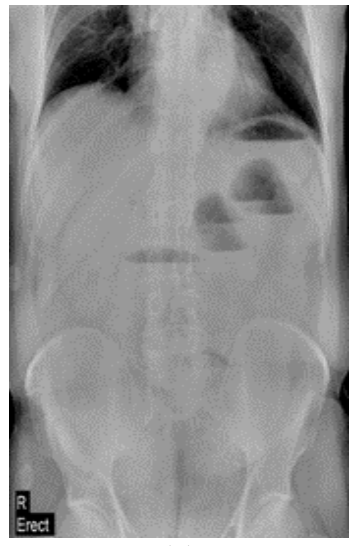

(a)

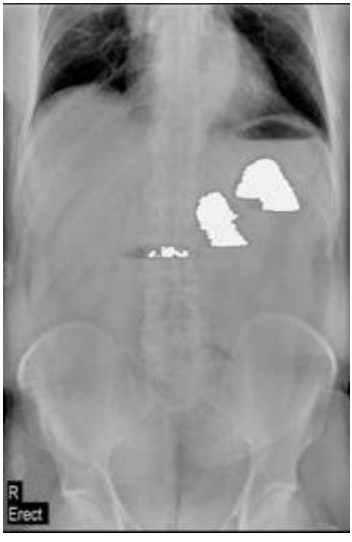

(b)

Figure 5. Examples of successful LBO feature extractions, (a) input, (b) successful extraction

\section{CONCLUSION}

In this paper, we propose a method to extract LBO feature (air-fluid area) automatically from Erect radiograph images. Erect abdominal radiography is better than supine abdominal radiography in inter- and intra-operator agreement but still this plain radiographic modality has operator subjectivity problem. Thus, we provide a fully automatic segmentation tool for doctors having objective evidences in diagnosis. The proposed method consists of many image processing procedures but the main engines for automatic

Automatic segmentation of large bowl obstruction area with Hough transform ... (Kwang Baek Kim) 
segmentation are Canny edge detector and Hough transform to locate air-fluid area from the input radiographic image. In experiment, the proposed method is perfect in automatic segmentation of target LBO feature when the image actually contains that feature(air-fluid region of sufficient size). There was no failed case among given 30 patients' images. However, this method has some limitations. Firstly, we test only real LBO patient cases. Thus, the $100 \%$ performance of this experiment should be read as true positive rate (TPR) only. Secondly, since we use morphological knowledge of LBO (edge length) in segmentation, the same procedure does not work properly in SBO segmentation. The main advantage of this approach is simple but strong objective tool for real-world pathologist in very specific limited usage.

\section{ACKNOWLEDGEMENTS}

This research was supported by the MSIT (Ministry of Science and ICT), Korea, under the Grand Information Technology Research Center support program (IITP-2020-0-01791) supervised by the IITP (Institute for Information \& communications Technology Planning \& Evaluation)

\section{REFERENCES}

[1] R. M. Gore, R. I. Silvers and K. H. Thakrar, et al., "Bowel Obstruction," Radiologic clinics of North America, vol. 53, no. 6, pp. 1225-1240, 2015.

[2] S. M. Bahouth, "Mechanical Obstruction: Large Bowel Obstruction (LBO)," Essential Radiology Review, pp. 289-290, 2019.

[3] T. Jaffe and W. M. Thompson, "Large-bowel obstruction in the adult: classic radiographic and CT findings, etiology, and mimics," Radiology, vol. 275, no. 3, pp. 651-663, 2015.

[4] R. M. Gore and M. S. Levine, "Textbook of gastrointestinal radiology," 3rd ed. Philadelphia, Pa: Saunders/Elsevier, 2008.

[5] P. Taourel, N. Kessler, A. Lesnik, J. Pujol, L. Morcos, and J. M. Bruel, "Helical CT of large bowel obstruction," Abdominal Imaging, vol. 28, no. 2, pp. 267-275, 2003.

[6] J. Welch, "Bowel obstruction: differential diagnosis and clinical management," Philadelphia, Pa: Saunders, 1989.

[7] R. S. Sawai, "Management of colonic obstruction: a review," Clinics in Colon and Rectal Surgery, vol. 25, no. 4, pp. 200-203, 2012.

[8] W. M. Thompson, R. K. Kilani, and B. B. Smith, et al., "Accuracy of abdominal radiography in acute small-bowel obstruction: does reviewer experience matter?," American Journal of Roentgenology, vol. 188, no. 3, pp. W233-W238, 2007.

[9] M. S. Cappell and M. Batke, "Mechanical obstruction of the small bowel and colon," The Medical Clinics of North America, vol. 92, no. 3, pp. 575-597, 2008.

[10] L. Plastaras, L. Vuitton, N. Badet, et al., "Acute colitis: Differential diagnosis using multidetector CT," Clinical Radiology, vol. 70, no. 3, pp. 262-269, 2015.

[11] T. Jess, M. Gamborg, P. Matzen, et al., "Increased risk of intestinal cancer in Crohn's disease: A meta-analysis of population-based cohort studies," The American Journal of Gastroenterology, vol. 100, no. 12, pp. 2724-2729, 2005.

[12] C. Duffin, S. Mirpour, T. Catanzano, C. Moore, "Radiologic Imaging of Bowel Infections," in Seminars in Ultrasound, CT and MRI, WB Saunders, vol. 41, no. 1, pp. 33-45, 2020.

[13] S. Ramanathan, V. Ojili, R. Vassa, A. Nagar, "Large bowel obstruction in the emergency department: imaging spectrum of common and uncommon causes," Journal of clinical imaging science, vol. 7, no. 15, 2017.

[14] J. D. Patel, H. I. Gale, K. J. Chang, "Imaging of Large Bowel with Multidetector Row CT," in Multislice CT, pp. 641-665, 2017.

[15] S. Suri, S. Gupta, and P. J. Sudhakar, et al., "Comparative evaluation of plain films, ultrasound and CT in the diagnosis of intestinal obstruction," Acta radiologica, vol. 40, no. 4, pp. 422-428, 1999.

[16] P. M. G. Alves, R. Monteiro, M. D. Claudino, A. R. Ventosa, R. Martins, and A. L. Moreira, "Large bowel obstruction: from suspicion to diagnosis," Presented at European Congress of Radiology, 2019.

[17] C. J. Das, S. Manchanda, A. Panda, A. Sharma, and A. K. Gupta, "Recent advances in imaging of small and large bowel," PET clinics, vol. 11, no. 1, pp. 21-37, 2016.

[18] K. B. Kim, D. H. Song, and S. S. Yun, "Automatic segmentation of wrist bone fracture area by K-means pixel clustering from X-ray image," International Journal of Electrical \& Computer Engineering (IJECE), vol. 9, no. 6, pp. 5205-5210, 2019.

[19] L. Park, D. H. Song, and H. Nho, et al., "Automatic segmentation of brachial artery based on fuzzy C-means pixel clustering from ultrasound images," International Journal of Electrical and Computer Engineering (IJECE), vol. 8, no. 2, pp. 638-644, 2018.

[20] W. Z. Geng, M. Fuller, B. Osborne, and K. Thoirs, "The value of the erect abdominal radiograph for the diagnosis of mechanical bowel obstruction and paralytic ileus in adults presenting with acute abdominal pain," Journal of Medical Radiation Sciences, vol. 65, no. 4, pp. 259-266, 2018.

[21] C. B. B. Lim, V. Che, A. Barsam, J. Berg J, and R. A. Harrison, "Plain abdominal radiographs: Can we interpret them?," Annals of the Royal College of Surgeons of England, vol. 88, no. 1, pp. 23-26, 2006.

[22] R. O. Duda and P. E. Hart, "Use of the Hough transformation to detect lines and curves in pictures," Communication of ACM, vol. 15, pp. 11-15, 1072. 
[23] A. Kalra and R. L. Chhokar, "A Hybrid approach using sobel and canny operator for digital image edge detection," 2016 International Conference on Micro-Electronics and Telecommunication Engineering (ICMETE), Ghaziabad, 2016, pp. 305-310.

[24] H. M. Ünver, Y. Kökve, E. Duman, and O. A. Erdem, "Statistical edge detection and circular Hough transform for optic disk localization," Applied Sciences, vol. 9, no. 2, pp. 350, 2019.

[25] R. K. Hapsari, M. I. Utoyo, R. Rulaningtyas, and H. Suprajitno, "Iris segmentation using Hough Transform method and Fuzzy C-Means method," Journal of Physics: Conference Series, vol. 1477, pp. 22-37, 2020.

[26] M. Abdullah, M. M. Fraz, S. A. Barman, "Localization and segmentation of optic disc in retinal images using circular Hough transform and grow-cut algorithm," PeerJ-Peer-Reviewed and Open Access, vol. 4, p. e2003, 2016.

[27] R. Vijayarajeswari, P. Parthasarathy, S. Vivekanandan, A. A. Basha, "Classification of mammogram for early detection of breast cancer using SVM classifier and Hough transform,” Meaqsurements, vol. 146, pp. 800-805, 2019.

\section{BIOGRAPHIES OF AUTHORS}

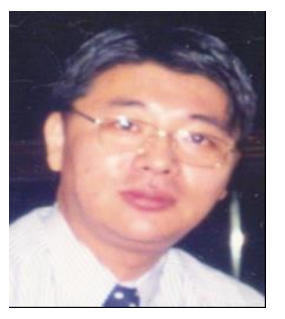

Kwang Baek Kim received his M.S. and Ph.D. degrees from the Department of Computer Science, Pusan National University, Busan, Korea, in 1993 and 1999, respectively. From 1997 to the present, he is a professor at the Division of Computer and Information Engineering, Silla University, Korea. He is currently an associate editor for Journal of Intelligence and Information Systems and The Journal of Information and Communication Convergence Engineering. His research interests include artificial intelligence, fuzzy clustering, data mining, image processing, and bioinformatics. He is currently the chief editor of Journal of Information and Communication Convergence Engineering.

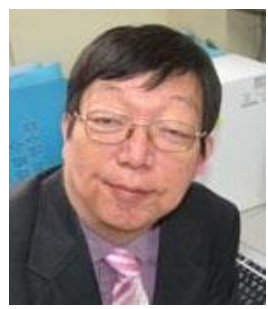

Doo Heon Song received his B.S. from Seoul National University in 1981 and M.S. from the Korea Advanced Institute of Science and Technology in 1983 in Computer Science. He received his Ph.D. candidate Certificate in Computer Science from the University of California at Irvine in 1994. He has been a professor at Department of Computer Games, Yong-in Songdam College, Korea, since 1997. His research interests include machine learning, artificial intelligence and computer game design. He is currently the associate editor of Journal of Information and Communication Convergence Engineering and Journal of Information Convergence and Communication Engineering.

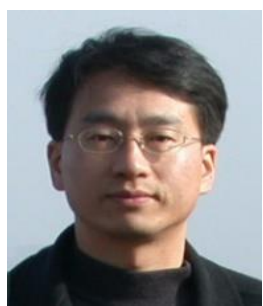

Young Woon Woo received his B.S. degree, M.S. degree and Ph.D. degree in electronic engineering from Yonsei University, Seoul, Korea in 1989, 1991 and 1997, respectively. Since 1997, he has been a professor in Department of Applied Software Eng., Dong-eui University, Busan, Korea. His research interests are in the area of artificial intelligence, image processing, pattern recognition and medical information. He is currently the president of Korea Institute of Information and Communication Engineering (KIICE). 